\title{
A Prospective Study Comparing Quality of Life and Cosmetic Results between Single-Port and Conventional Laparoscopic Cholecystectomy
}

\author{
Olaf Teubner' ${ }^{1}$, Claus D. Heidecke1, Thomas Kohlmann², Kaja Ludwig3, Maciej Patrzyk ${ }^{1 *}$ \\ ${ }^{1}$ Department of Surgery, Clinic of General Surgery, Visceral, Thoracic and Vascular Surgery, \\ Ernst-Moritz-Arndt-University Hospital, Greifswald, Germany \\ ${ }^{2}$ Institute for Community Medicine, Ernst-Moritz-Arndt-University, Greifswald, Germany \\ ${ }^{3}$ Department of Surgery, Südstadt Hospital, Rostock, Germany \\ Email: patrzyk@uni-greifswald.de
}

Received 22 January 2016; accepted 22 February 2016; published 25 February 2016

Copyright $@ 2016$ by authors and Scientific Research Publishing Inc.

This work is licensed under the Creative Commons Attribution International License (CC BY). http://creativecommons.org/licenses/by/4.0/

\begin{abstract}
Background: Multiple studies from the last five years have demonstrated that single-incision laparoscopic surgery cholecystectomy (SILS) is not only feasible but also produces a result comparable with that of conventional laparoscopic cholecystectomy (CLC). Methods and results: In this bicentric study, we used the Short Form (36) (SF-36) and Nottingham Health Profile (NHP) surveys to estimate and compare the post-operative quality of life up to the 28th post-operative day for 66 patients who had undergone either CLC $(n=32)$ or SILS $(n=34)$. Additionally, we investigated patient satisfaction with the cosmetic results after one year. The curves summarizing the eight sections in the physical sum scale and in the mental sum scale (SF-36) were the same within the confidence interval with a confidence level of $95 \%$. Thus, post-operative quality of life could be assumed to be equivalent for the two groups. Evaluation of the NHP survey produced similar results. The perceived cosmetic results were significantly better for the SILS group (1.3 on a scale of 1 - 5 with 1 being the best) than for the CLC group (1.9) $(p=0.016)$. Conclusions: This study did not demonstrate better quality of life for the single-port procedure as it had been expected. Instead, the single-port procedure produced subjectively better cosmetic results.
\end{abstract}

\section{Keywords}

Single-Port Laparoscopic Cholecystectomy, Conventional Laparoscopic Cholecystectomy, Quality

\footnotetext{
${ }^{*}$ Corresponding author.
}

How to cite this paper: Teubner, O., Heidecke, C.D., Kohlmann, T., Ludwig, K. and Patrzyk, M. (2016) A Prospective Study Comparing Quality of Life and Cosmetic Results between Single-Port and Conventional Laparoscopic Cholecystectomy. Surgical Science, 7, 114-125. http://dx.doi.org/10.4236/ss.2016.72016 


\section{Introduction}

The gold standard in cholecystectomies today is the laparoscopic procedure. Currently, more than 95\% of all cholecystectomies are performed in this manner. This development is driven by the desire to improve the quality of life by minimizing tissue damage. In the search for new means of optimizing laparoscopy, single-port techniques point in the direction of scar-free surgery. The first discussions and case studies on the subject appeared in 2008. In the next two years, various German centres began trying the new procedures. But by 2010, the heyday of single-port cholecystectomies had passed. The aims of many studies were to assess morbidity and patient-specific outcomes after single-incision laparoscopic surgery (SILS) and to make comparisons, where possible, with conventional laparoscopic cholecystectomy (CLC) [1]-[6]. A further all-encompassing spread of this technique as had happened with laparoscopy during the early 1990s, never occurred. In certain surgical centres, however, single-port procedures had shifted from gall bladder surgery to colon surgery. In discussions and publications on single-port procedures, another, previously ignored aspect was beginning to take centre stage: the cosmetic result. The possibility of performing surgery without leaving any visible scar constituted a truly new degree of surgical quality. At this point, it was important to determine the extent to which these new surgical methods fulfilled the expectations of a better quality of life (due to less pain and faster recovery) compared to conventional laparoscopy and also how strong the patients' interest was in the demonstrably superior cosmetic result. The goal of this prospective study was to compare the post-operative quality of life after elective cholecystectomies performed with conventional laparoscopic methods and with single-port methods. With regard to quality of life, three aspects were investigated: satisfaction with the cosmetic result, general quality of life based on the SF-36 survey, and the Nottingham Health Profile (NHP).

\section{Materials and Methods}

The design of this study is prospective and bicentric. We investigated 66 patients who were operated on between May 2010 and June 2011 in a municipal maximum-care hospital (24 patients) or in a university hospital (42 patients). The surgical diagnosis was symptomatic cholecystolithiasis, and each patient, as requested, was assigned to either the conventional laparoscopy group (with four trocar incisions; $2 \times 5 \mathrm{~mm}$ and 2x12 mm ports) or to the single-port group (one umbilical incision; $20 \mathrm{~mm}$ ) according to patient preference. All single-port operations were performed using a SILS-Port ${ }^{\mathrm{TM}}$ with $2 \times 5 \mathrm{~mm}$ and $1 \times 12 \mathrm{~mm}$ ports (Covidien, Neustadt a.d, Germany). Patient names were replaced with pseudonyms before inclusion in the study. After the surveys were returned, the data were also processed using the pseudonyms so that no information obtained could be matched to actual patient names. Preoperatively, all patients received a questionnaire on the quality of life, based on the SF-36 and the Nottingham Health Profile. Patients then made entries each day, beginning preoperatively and then subsequently on the first day after surgery and continuing until the 28th post-operative day.

The cosmetic result was determined based on the subjective optical evaluation of the abdominal operative field. The survey on cosmetic results took place one year after surgery. Participants were asked to rate the cosmetic result on a scale from 1 to 5 , with 1 being the best. No comparison between the two surgical procedures occurred. Each patient was asked to rate their own result alone.

Patients included in this study had symptomatic cholecystolithiasis with stones of $<3 \mathrm{~cm}$. Patient age was restricted to 18 - 75 years, and patients were required to have a BMI of $40 \mathrm{~kg} / \mathrm{m}^{2}$ or less and an ASA score lower than IV. We excluded patients younger than 18, older than 75 , with BMIs $>40 \mathrm{~kg} / \mathrm{m}^{2}$, or ASA IV-V. We also excluded patients with gallstones larger than $3 \mathrm{~cm}$, choledocholithiasis, acute or chronic cholecystitis, and coagulopathies including thrombocytopenia of $<60,000 / \mathrm{ml}$.

Exclusion criteria also included severe alcoholism, neuromuscular disorders, analgesic abuse, pregnancy, any conditions where pneumoperitoneum is contraindicated, and lack of patient willingness to participate in the study. Thirty-four patients were assigned to the single-port group (group B/SILS) and 32 to the conventional laparoscopy group (group A/CLC) (Table 1).

Among the study participants, $72.7 \%$ were female $(n=48)$, and $27.3 \%$ were male $(n=18)$. Group A (CLC) was composed of $62.5 \%$ women $(n=20)$ and $37.5 \%$ men $(n=12)$, while group B (SILS) included $82.4 \%$ wom- 
en $(n=28)$ and $17.6 \%$ men $(n=6)$. The gender distribution in our study corresponds to the general gender distribution for gall bladder patients. The average age of the study participants was 53.8. The youngest patient was 20, and the oldest was 75. In group A (CLC), the average age was 59 (range: 20 - 74), and in group B (SILS), the average age was 48.8 (range: 24 - 75). Since cosmetic satisfaction is a special criterion, which is likely correlated to age, we compared the age group distribution in the image below (Figure 1).

The average BMI was $27.3 \mathrm{~kg} / \mathrm{m}^{2}$. The distribution was relatively uniform with an average BMI of $27.5 \mathrm{~kg} / \mathrm{m}^{2}$ (range: 20.0 - 39.0) in group A (CLC) and $27.2 \mathrm{~kg} / \mathrm{m}^{2}$ (range: 20.1 - 32.9) in group B (SILS). The t-test produced a p-value of $\mathrm{p}=0.728$, meaning that no statistically significant difference exists. The median ASA was II in both groups (see Table 2).

Quality of life was determined based on the SF-66 survey and the Nottingham Health Profile. The "Medical Outcomes Study 36-item Short Form Health Survey" (MOS-SF-36) contains 36 questions. The answer to each question (item) corresponds to a specific, predetermined item value. The 36 items are distributed into eight dif-

\begin{tabular}{cccccc}
\multicolumn{2}{l}{ Table 1. Distribution of study participants. } \\
\cline { 3 - 5 } & & \multicolumn{2}{c}{ Hospital } & \multirow{2}{*}{ Total } \\
\cline { 3 - 5 } & & University hospital & Municipal hospital & \\
\hline \multirow{3}{*}{ Operation } & CLC & Number & 20 & 12 & 32 \\
& & $\%$ & $47.6 \%$ & $50.0 \%$ & $48.5 \%$ \\
& SILS & Number & 22 & 12 & 34 \\
& & $\%$ & $52.4 \%$ & $50.0 \%$ & $51.5 \%$ \\
\hline \multirow{2}{*}{ Total } & & Number & 42 & 24 & 66 \\
& & $\%$ & $100.0 \%$ & $100.0 \%$ & $100.0 \%$ \\
\hline
\end{tabular}

Table 2. Distribution of ASA classification in both groups.

\begin{tabular}{|c|c|c|c|c|c|c|}
\hline & & & \multicolumn{3}{|c|}{ ASA } & \multirow{2}{*}{ Total } \\
\hline & & & I & II & III & \\
\hline \multirow{4}{*}{ Operation } & \multirow{2}{*}{ CLC } & Number & 4 & 22 & 6 & 32 \\
\hline & & $\%$ & $40.0 \%$ & $44.9 \%$ & $85.7 \%$ & $48.5 \%$ \\
\hline & \multirow{2}{*}{ SILS } & Number & 6 & 27 & 1 & 34 \\
\hline & & $\%$ & $60.0 \%$ & $55.1 \%$ & $14.3 \%$ & $51.5 \%$ \\
\hline \multirow{2}{*}{\multicolumn{2}{|c|}{ Total }} & Number & 10 & 49 & 7 & 66 \\
\hline & & $\%$ & $100.0 \%$ & $100.0 \%$ & $100.0 \%$ & $100.0 \%$ \\
\hline
\end{tabular}

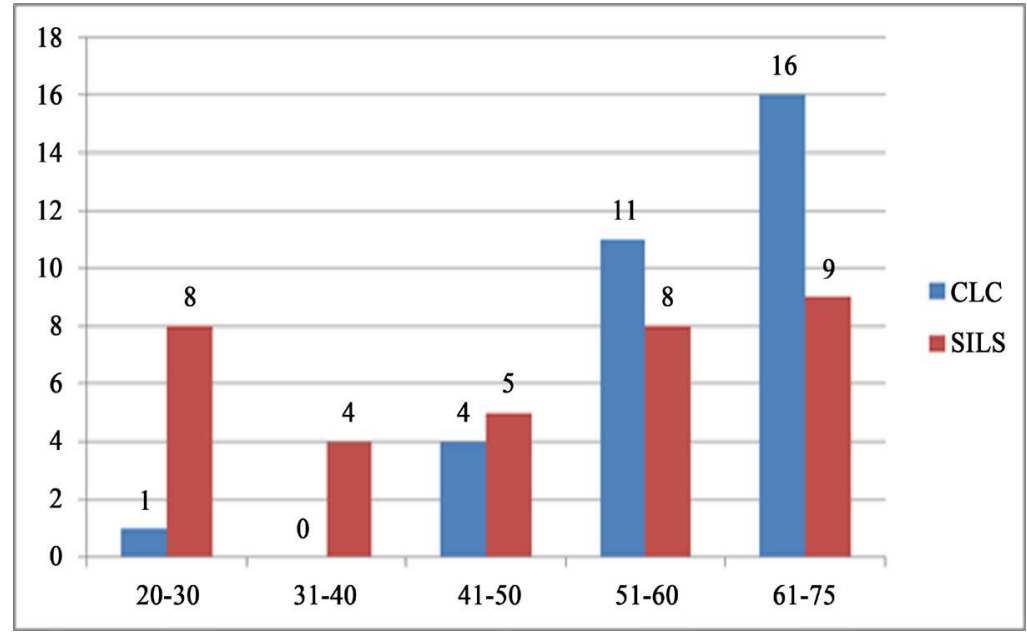

Figure 1. Distribution according to age. 
ferent scales. Each scale measures a different aspect of subjective patient health. The scales are coded with a scale value, which is based on the item value of each scale. The individual scale value is thus dependent on the answers to one or more questions. Higher scale values correspond to better patient quality of life. This means that for the pain section, for example, zero corresponds to maximum pain and 100 to minimum pain. The eight sections are physical functioning, physical role functioning, bodily pain, general health perceptions, vitality, social role functioning, emotional role functioning, and mental health. The eight sections are combined in the mental and physical composite scores. The physical composite includes the subscales "physical functioning", "physical role functioning", "bodily pain", and "general health perceptions". The mental composite includes "social role functioning", "emotional role functioning", and "mental health".

The Nottingham Health Profile (NHP) is one of the most frequently used tools for measuring subjective health and health-related quality of life. The NHP investigates perceived feelings and emotions. The focus is more on how patients perceive their state of health than on their health-related behaviour. The NHP survey consists of 38 items that are separated into six sections: energy level (3 items), pain (8 items), emotional reaction (9 items), sleep (5 items), social isolation (5 items), and physical abilities (8 items). The questions were answered on the same days as the questions from the SF-36 survey: from the first to the 28th post-operative day. This study was approved by the ethics committee of the Ernst-Moritz-Arndt Medical University of Greifswald.

\section{Statistical Evaluation}

All of the surveys sent out were returned. The individual scale scores for each patient were calculated using the computer program SPSS Version 20.0. In the statistical analysis, we compared the eight section scores (dependent variables) on patient quality of life, as related to certain factors (independent variables) such as gender and age group. Initially, we tested the null hypothesis (H0: "The average vector of quality of life is the same in all levels (categories) of independent factors"). Since this is an orienting analysis, we selected the following for quality of life:

1) For each recording time, the differences between the groups were analysed. We then performed an orienting unpaired t-test. The changes over time are presented in diagrams.

2) We calculated the areas under the curves in order to compare the complete courses from the first to the 28th post-operative day.

3) We controlled for further variables such as age, gender, and ASA score using a linear regression analysis.

A level of $5 \%$ was set for all tests of significance, meaning that the null hypothesis was rejected for all p-values of $\leq 0.05$.

\section{Results}

\subsection{Quality of Life Results Based on SF-36}

The images below show the section scores, compared to the US American standard values, plotted over time on the $y$-axis. In this representation, 100 is the maximum score. The $\mathrm{x}$-axis depicts the eight recording times, from the first to the 28th post-operative day.

The physical summed score represents a composite of the scores for the individual sections (physical functioning, physical role functioning, bodily pain, and general health perceptions) compared to the standard sample values. The values plotted on the curve, and thus the course of the curve, are nearly identical. The two surgical procedures yield the same physical summed score (Figure 2(a)). The bodily pain section is particularly interesting in terms of the comparison between the two procedures, as here it deviates from the other sections included in the physical composite score (Figure 2(b)). The y-axis scales the comparable standard values, where 0 corresponds to extreme pain and 100 corresponds to total absence of pain. From post-operative day one to postoperative day 10 the pain rating is higher for the SILS group than for the CLC group. For the first post-operative day, this difference is statistically significant $(\mathrm{p}=0.043)$. The separate recording times show different values; they cannot, however, be viewed in isolation from the overall progression of the curve, meaning that the differences lie within the tolerance in the predetermined confidence interval (confidence level 95\%) and are thus not statistically significant. The mental summed score represents the composite of the vitality, social functioning, emotional role functioning, and mental health sections compared to the standard values on the y-axis. The values represented on the curve and thus the curve progression are nearly the same. The difference for the first post-operative day is not significant $(\mathrm{p}=0.155)$. The mental composite score for the two groups is thus the same 

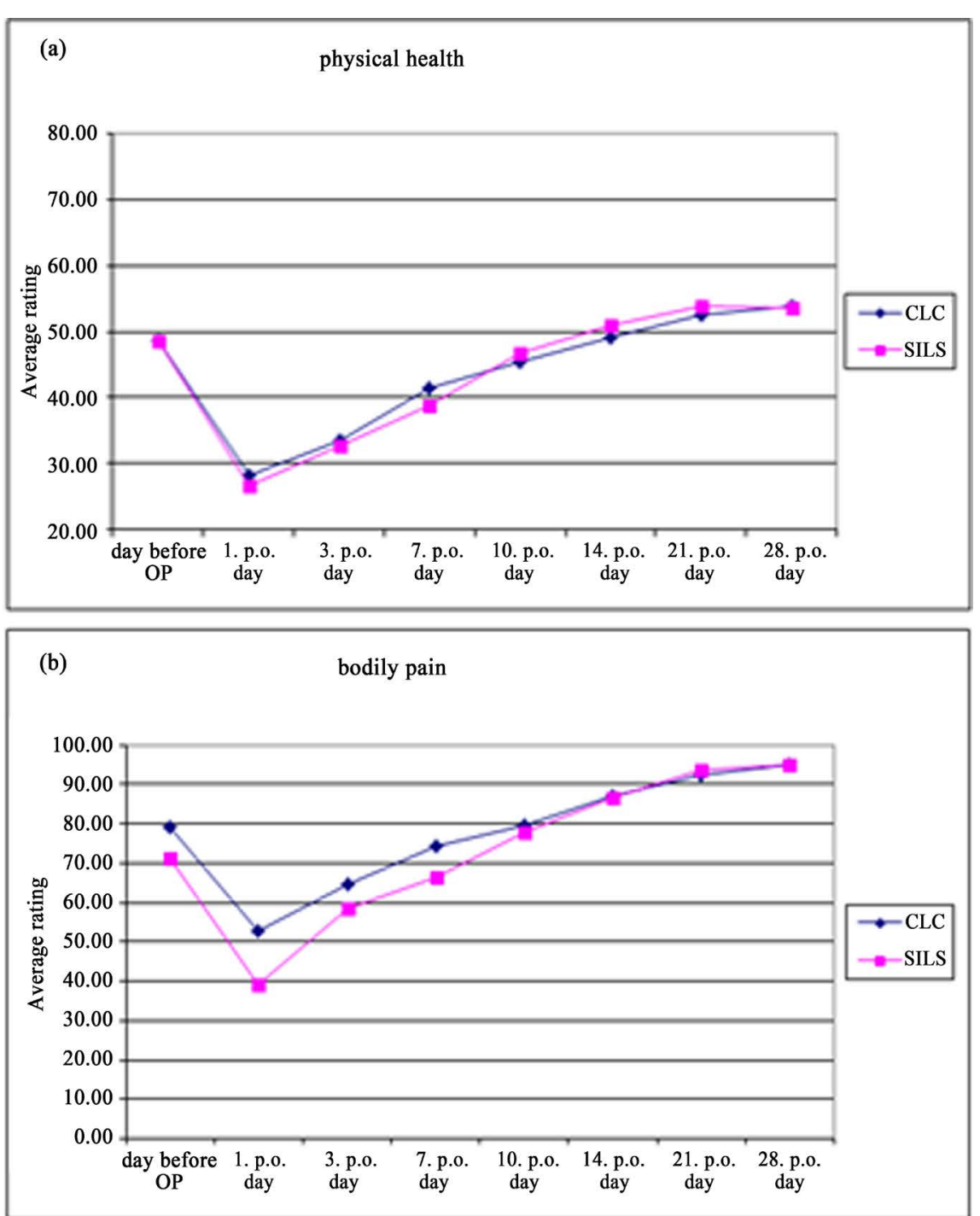

(c)

mantal health

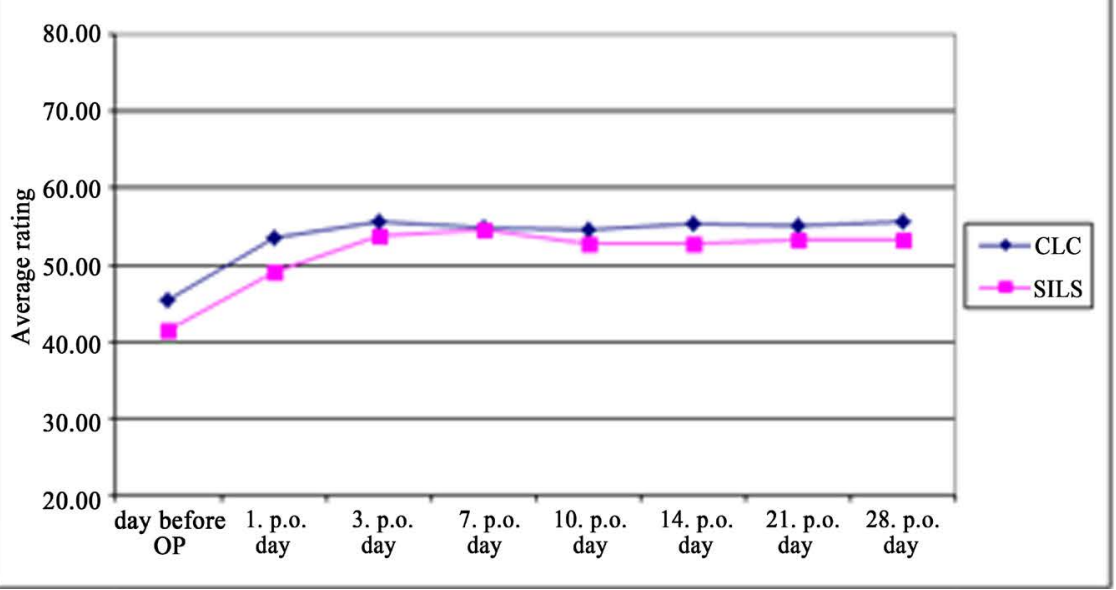

Figure 2. Quality of life beginning preoperatively and continuing until the 28th post-operative day after SILS and CLC based on SF-36. (a) Physical summed score, (b) Bodily pain, (c) Mental composite scale. 
(Figure 2(c)).

\subsection{Regression Analysis}

As reported in the methods, there were some partially relevant differences in age, gender, operation time, and ASA classification. Our interest was in determining whether these differences had an impact on the result. In order to control for the differences in age, gender, and ASA status, we performed a multiple linear regression analysis. The dependent variables formed the areas under the curve of the eight subscores and the two composite scores of the SF-36. Additionally, the three control variables were incorporated as predictors into the linear regression model. There was no statistically significant result for any of the adjusted therapy effects (all p-values > $0.4)$.

\subsection{Quality of Life Results Based on the Nottingham Health Profile}

On the y-axis these images depict the curve progression of the measured values in comparison to the US American standard values. It should be noted that these values are also scaled from 0 to 100 , but here a lower score corresponds to a better result (a healthier patient). On the x-axis, the eight measurement times are recorded, from the first to the 28th post-operative day.

The energy level differences between the groups were at all times $\mathrm{p}>0.05$ (between $\mathrm{p}=0.293$ and $\mathrm{p}=0.742$ ) and thus not significant (Figure 3(a)). In terms of pain, the difference between the groups (on post-operative day 1) has a p-value of 0.032 and is thus statistically significant (Figure 3(b)). In other words, the SILS group had more pain on the first post-operative day. By the third post-operative day, however, the difference was no longer significant $(p=0.154)$. The values from the individual recorded points in time cannot be viewed in isolation from the total curve progression, meaning that the difference over time is within the range of tolerance (in a predetermined confidence interval with a confidence level of 95\%) and that the total pain score is not statistically significant.

The initial difference in emotional reaction is somewhat larger on the day before surgery, but in the post-operative course, it is no longer significant ( $p>0.185$ ) and thus nearly identical for the two groups (Figure 3(c)).

In terms of the post-operative sleep score, the SILS group shows slightly better results (Figure 4(a)), but here too the p-values of 0.405 on the third post-operative day and $p=0.104$ on the 28th post-operative day indicate a non-significant difference. The social isolation category, with p-values of $p=0.246$ on the first post-operative day and $p=0.751$ on the 28th post-operative day, also has nearly identical scores (Figure 4(b)). For the physical abilities section, on the first post-operative day, the CLC group had a better result, which was also statistically significant. The remaining p-values (up to $\mathrm{p}=0.875$ ) are not significant. Here again, the overall course is what matters, and the scores and individual recording times cannot be considered separately (Figure 4(c)). The results from the NHP analysis are comparable to those from the SF-36.

\subsection{Satisfaction with the Cosmetic Result}

Patient satisfaction regarding the cosmetic result was based on a questionnaire sent one year after the operation in which patients gave a score of between 1 and 5 (with 1 being the best result). The SILS group did better with a score of 1.3, compared to 1.9 in the CLC group (Figure 5). The Independent Samples Test produced a p-value of 0.016 , confirming statistical significance.

There were two dramatic outliers with an extremely negative evaluation in the SILS group and one in the CLC group.

\section{Discussion}

Single-port cholecystectomy is often viewed as an intermediary step between conventional laparoscopy and the "scar-free" Natural Orifice Transluminal Endoscopic Surgery (NOTES) procedure. The hope for this intermediary procedure was that it would mean less tissue damage, a better outcome for patients, a better quality of life, and a better cosmetic result. The implementation of NOTES procedures and thus the development of new surgical instruments in this realm are stagnating, however, which raises the question of whether the single-port procedure taken by itself might become the next qualitative step in surgical practice. In this regard, scientific evi- 

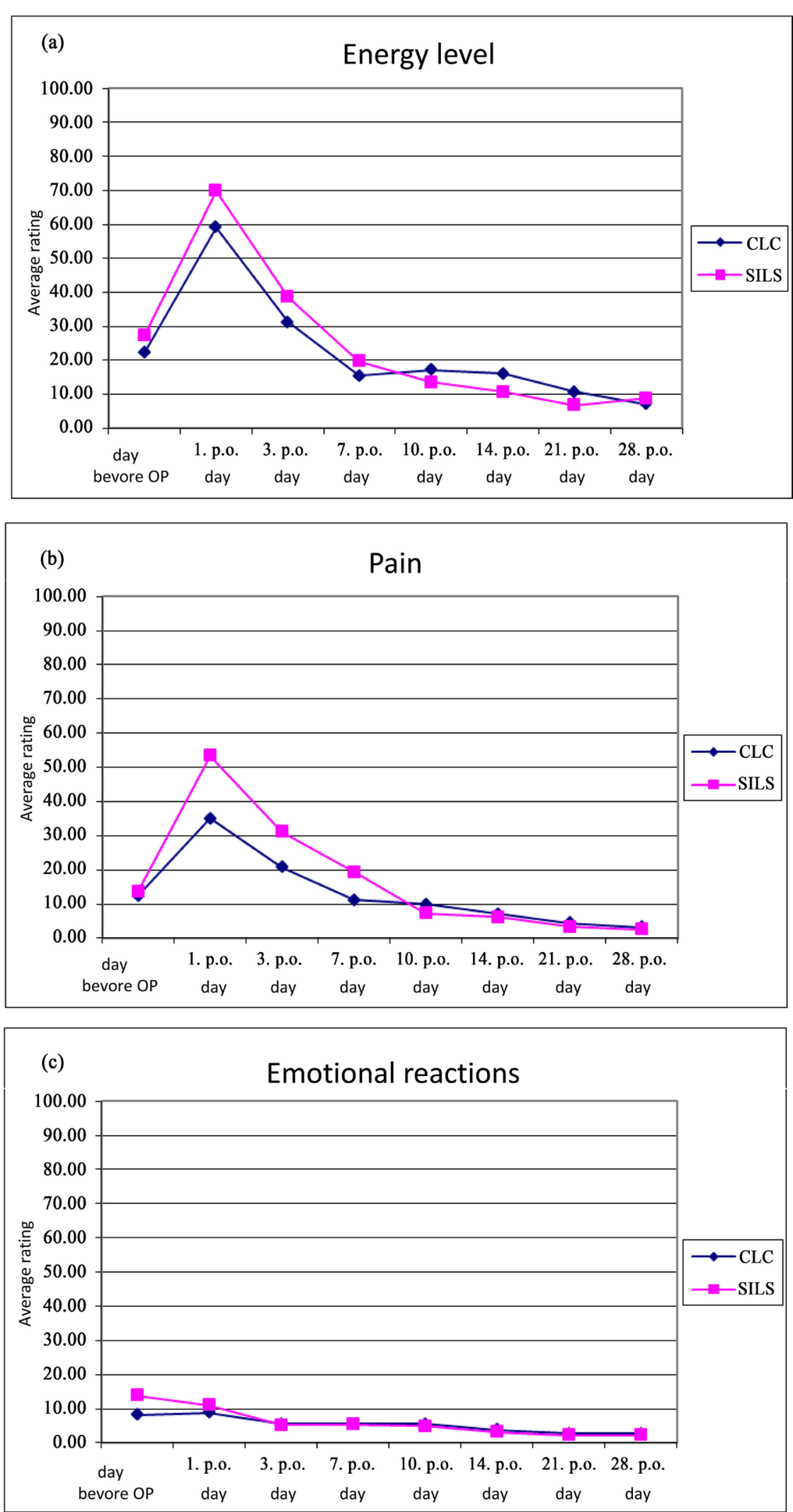

Figure 3. Quality of life beginning preoperatively and continuing until the 28th post-operative day after SILS and CLC based on the Nottingham Health Profile. (a) Energy level subsection. (b) Pain subsection. (c) Emotional reaction subsection. 

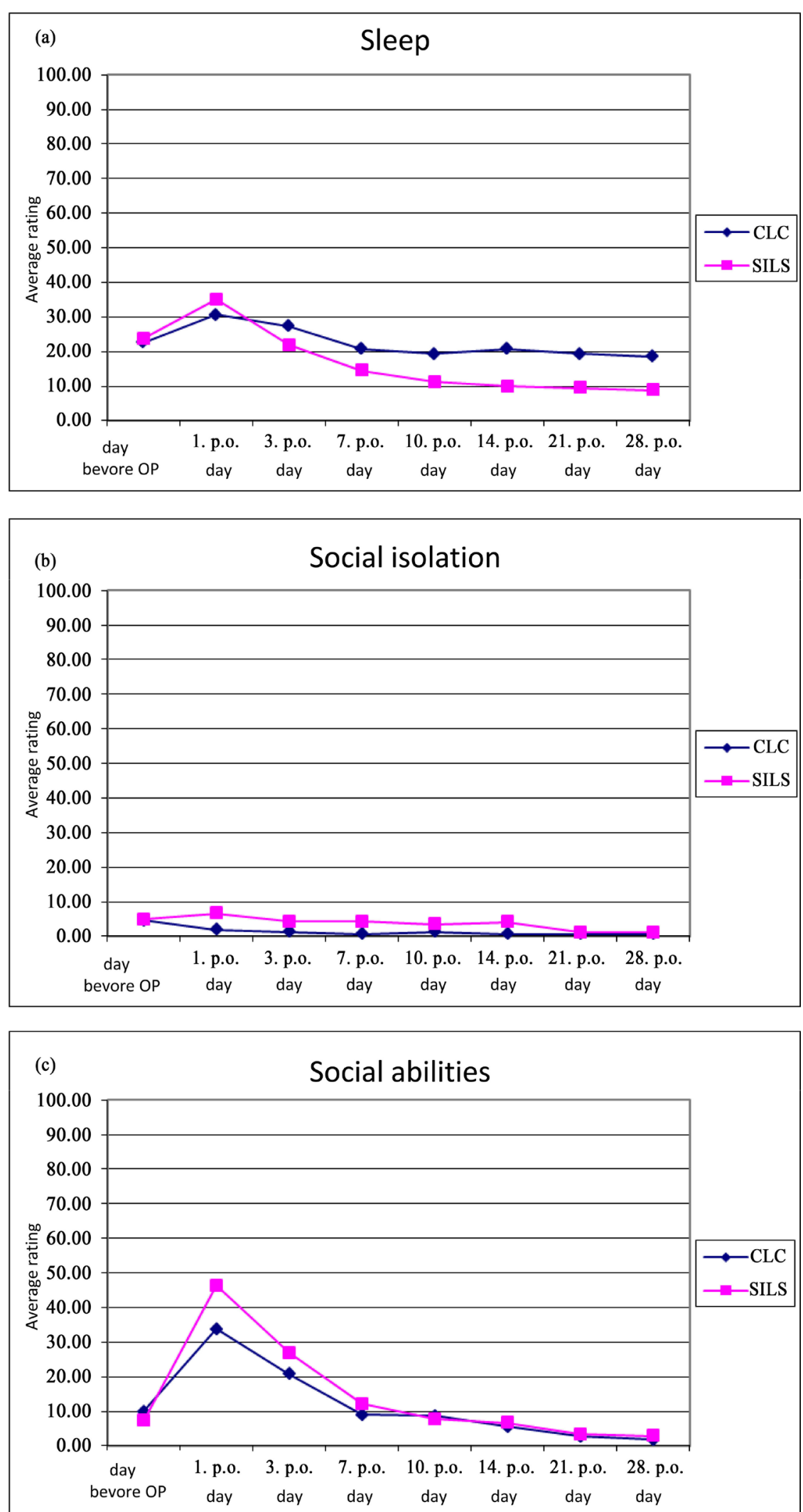

Figure 4. Quality of life beginning preoperatively and continuing until the 28th post-operative day after SILS and CLC based on the Nottingham Health Profile (a) Sleep subsection. (b) Social isolation subsection. (c) Physical abilities subsection. 


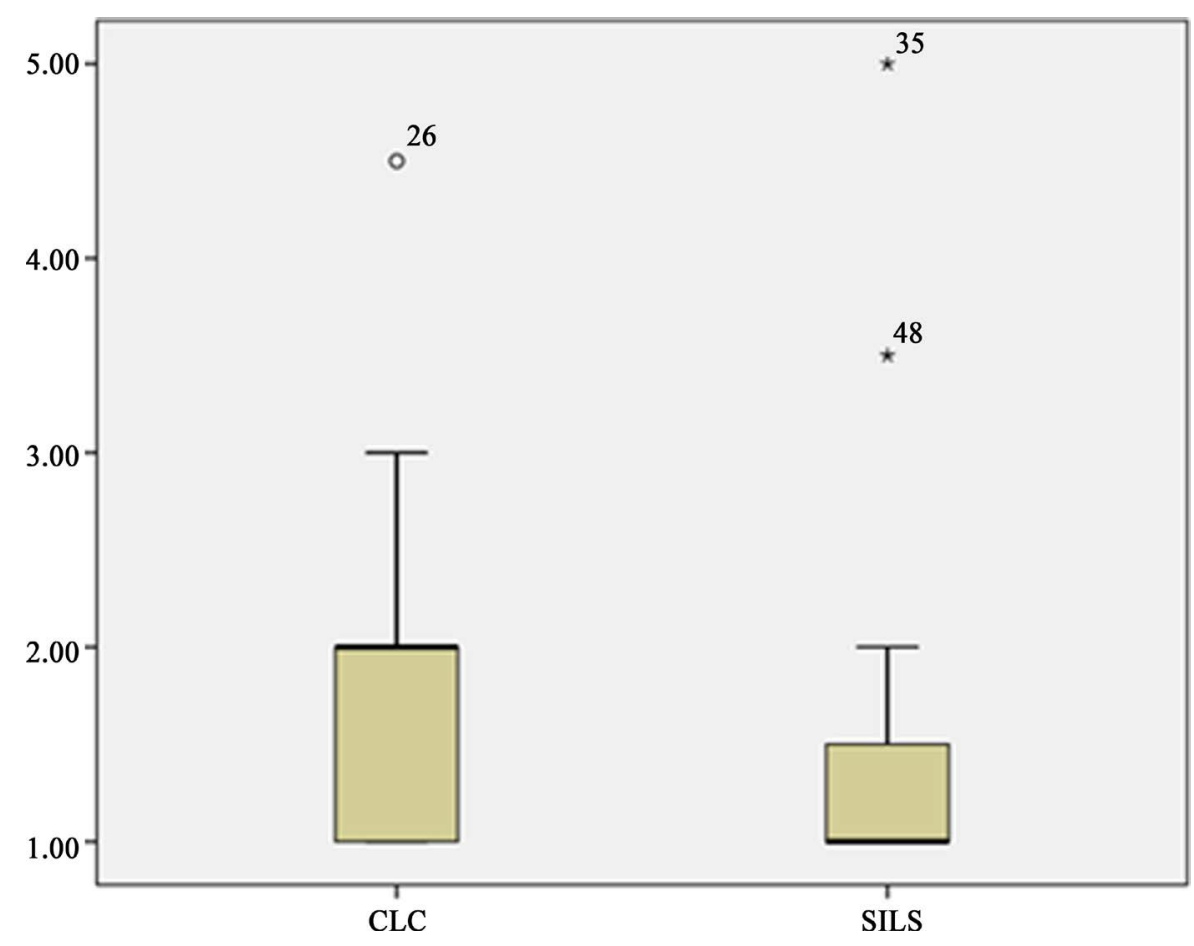

Figure 5. Cosmetic result: CLC vs. SILS (evaluation on a handy school grading system scale: 1 -very good and 5 -inadequate).

dence is required to demonstrate not just comparability but also superiority in at least one aspect.

There are currently few studies that use a standardized measurement tool to investigate the broad criteria necessary for a quality of life comparison [7] [8].

This study included 66 patients, of whom 32 underwent CLC and 34 underwent SILS, a relatively uniform distribution. The novelty of this surgical procedure makes a significantly larger sample size difficult, and our study numbers are comparable with those of most existing published studies. To facilitate comparison with existing data and also to minimize external risk factors, we based our inclusion and exclusion criteria as much as possible on already published studies. A percentage of $72.2 \%$ of our study participants were female. This reflects the gender distribution of gall bladder disease among the population at large. The average age of our study participants also reflects the broader population of gall bladder patients. The BMI of $27 \mathrm{~kg} / \mathrm{m}^{2}$, which was identical in the two groups, also corresponds to the broader patient population as reflected in existing studies [9] where the average ranges from 25 to $28 \mathrm{~kg} / \mathrm{m}^{2}$. The median ASA in both our groups was II, although an ASA of III was not an exclusion criterion, as it is in a few published studies [10]-[12]. We considered age, gender, operation time, and ASA classification separately and performed multiple linear regression analyses in order to determine whether these factors had an impact on the composite physical or mental scores. We determined that none of these factors had a statistically significant effect on the results presented in this paper (all p-values were > 0.4). The individual items of SF-36 were mathematically aggregated into eight sections and then grouped into the composite physical score and the composite mental score. In seven of the eight sections, the curve progressions were essentially identical for the two groups. There was, however, a noticeable difference in the pain rating. From the first post-operative day to the first post-operative week, the SILS group experienced more pain than the CLC group. Existing studies [7] [8] [10] [13]-[17] give pain scores, within the confidence interval with a confidence level of 95\%, that have no statistically significant difference at six and 24 hours or at one week after surgery. Within this tolerance, there is a variation in pain perception, and in more than half the studies, the SILS group reports slightly more pain. This matches the analysis presented in this study. For the first post-operative day, we saw a significant difference for both the NHP $(p=0.032)$ and for the SF-36 ( $=0.043)$. From post-operative day two to post-operative day 10, the pain ratings in the SILS group were higher but not statistically significant. From the 11th post-operative day on, the pain ratings were the same. This raises the question of whether the differences in pain rating are due to the different incision types. Garg et al. [10], as well as Blinman 
et al. [18], discuss this question. In comparisons between SILS and CLS, it seems that this is indeed the reason for the slightly higher pain ratings in the SILS group in the time period of six hours to one week after surgery. At the same time, however, the difference between the two groups is so small that it cannot even be considered statistically significant. Other published studies even describe the opposite result. The curves for the SILS and CLC groups are nearly the same for the "vitality" and "pain" sections. This would suggest that vitality is dependent on pain perception or is influenced by it. Similarly, we can assume that the slightly poorer "mental health" rating up until the third post-operative day in the SILS group is due to the perceived pain intensity. Since the curves summarizing the eight sections in the physical and mental composite scores within the confidence interval and with a confidence level of $95 \%$ are the same, we can assume that the two groups had the same level of post-operative quality of life. In 2013, Wagner et al. [7] published a prospective cohort study with 222 patients in which quality of life in particular was investigated using the EQ-5D-5L survey. In 2011, Marks et al. [8] published a prospective randomized study with 200 patients that examined the general post-operative quality of life based on the SF-12, the short form of the SF-36. Overall quality of life was also investigated by Leung et al. [19]. None of these studies found a statistically significant difference in the total score or in the deviation from the baseline $(\mathrm{p}>0.05)$.

The results of the Nottingham Health Profile offer the same conclusion, strongly supporting our analysis of the SF-36. There is a broad correlation between the commonalities. Unimportant differences can be ignored as they have p-values greater than 0.05 and are thus not significant. The additional NHP analysis gives clear proof of the statistical stability and power of the results. Deviations in the individual investigation criteria are for the most part below the threshold for statistical significance, and when they are registered, they usually give varying results. Since all criteria described until now suggest no clear advantage either for the surgical method, the most important difference is the individual perception of the cosmetic result. For the SILS group, the subjective perception of the cosmetic result was significantly better. Since satisfaction with the cosmetic result has a direct influence on the mental health of the patient, and since this in turn is a crucial aspect of quality of life, in this regard the SILS procedure is clearly superior. In nearly all published studies, the cosmetic result is statistically significantly better for the SILS group [8] [13] [14] [16] [17] [20]-[22]. The relevance of this demonstrated advantage of SILS should thus be decided on an individual basis. Both procedures should be offered, and it should then be left up to the patient to decide. The average age of their study participants was 54, which is indeed relatively high, but in light of the demographic changes as well as the changes in attitude regarding physical attractiveness in recent years, even for older patients the cosmetic advantage is growing to be more and more relevant. It is thus important to determine how the cosmetic effect influences patients' decisions to be operated on with the single-port procedure and also to determine how surgical departments can use this to their advantage for marketing themselves to patients.

There are however some limitations inherent to the study. Baseline demographical data and quality of life parameters need to be statistically compared for the same reasons. It should be considered that there are differences in ASA score and gender distribution between groups, which might have influenced both well-being perception (older v. younger patients), pain (younger v. older patients and females v. males) and satisfaction with the cosmetic results. The present study population is too small to detect such effects. Emerging evidence suggests a higher incidence of port-site hernia following SILS. The follow-up after more than one year would be necessary to detect this problem.

\section{Conclusion}

This study did not demonstrate better quality of life for the single-port procedure as it had been expected. The single-port technique offered subjectively a better cosmetic result for patients after cholecystectomy.

\section{Funding}

This study was not sponsored by a company or any institution

\section{Disclosures}

Drs. Claus D. Heidecke, Thomas Kohlmann, Kaja Ludwig, and Maciej Patrzyk have no conflicts of interest or financial ties to disclose. Dr. Olaf Teubner is a scientific assistant. 


\section{Ethical Approval}

All procedures performed in studies involving human participants were in accordance with the ethical standards of the institutional and/or national research committee and with the 1964 Helsinki declaration and its later amendments or comparable ethical standards. The study has been approved by the appropriate institutional research ethics committee.

\section{Informed Consent}

Informed consent was obtained from all individual participants included in the study.

\section{References}

[1] Romanelli, J.R. and Earle, D.B. (2009) Single-Port Laparoscopic Surgery: An Overview. Surgical Endoscopy, 23, 1419-1427. http://dx.doi.org/10.1007/s00464-009-0463-x

[2] Langwieler, T.E., Nimmesgern, T. and Back, M. (2009) Single-Port Access in Laparoscopic Cholecystectomy. Surgical Endoscopy, 23, 1138-1141. http://dx.doi.org/10.1007/s00464-009-0389-3

[3] Tsimoyiannis, E.C., Tsimogiannis, K.E., Pappas-Gogos, G., Farantos, C., Benetatos, N., Mavridou, P., et al. (2010) Different Pain Scores in Single Transumbilical Incision Laparoscopic Cholecystectomy versus Classic Laparoscopic Cholecystectomy: A Randomized Controlled trial. Surgical Endoscopy, 24, 1842-1848. http://dx.doi.org/10.1007/s00464-010-0887-3

[4] Marks, J., Tacchino, R., Roberts, K., Onders, R., Denoto, G., Paraskeva, P., et al. (2011) Prospective Randomized Controlled Trial of Traditional Laparoscopic Cholecystectomy versus Single-Incision Laparoscopic Cholecystectomy: Report of Preliminary Data. The American Journal of Surgery, 201, 369-373. http://dx.doi.org/10.1016/j.amjsurg.2010.09.012

[5] Bucher, P., Pugin, F., Buchs, N.C., Ostermann, S. and Morel, P. (2011) Randomized Clinical Trial of Laparoendoscopic Single-Site versus Conventional Laparoscopic Cholecystectomy. British Journal of Surgery, 98, 1695-1702. http://dx.doi.org/10.1002/bjs.7689

[6] van der Linden, Y.T., Bosscha, K., Prins, H.A. and Lips, D.J. (2015) Single-Port Laparoscopic Cholecystectomy vs Standard Laparoscopic Cholecystectomy: A Non-Randomized, Age-Matched Single Center Trial. World Journal of Gastrointestinal Surgery, 7, 145-151. http://dx.doi.org/10.4240/wjgs.v7.i8.145

[7] Wagner, M.J., Kern, H., Hapfelmeier, A., Mehler, J. and Schönberg, M.H. (2013) Single-Port Cholezystektomie versus Multi-Port Cholecystektomy: A Prospective Cohort Study with 222 Patients. World Journal of Surgery, 37, 991-998. http://dx.doi.org/10.1007/s00268-013-1946-4

[8] Marks, J., Tacchino, R., Roberts, K., Onders, R., Denoto, G., Paraskeva, P., Rivas, H., Soper, N., Rosemurgy, A. and Shah, S. (2011) Prospective Randomized Controlled Trial of Traditional Laparoscopic Cholecystectomy versus Single-Incision Laparoscopic Cholecystectomy: Report of Preliminary Data. The American Journal of Surgery, 201, 369372. http://dx.doi.org/10.1016/j.amjsurg.2010.09.012

[9] Garg, P., Deep, J.T., Garg, M. and Menon, G.R. (2012) Single-Incision Laparoscopic Cholecystectomy vs. Conventional Laparoscopic Cholecystectomy: A Meta-Analysis of Randomized Controlled Trials. Journal of Gastrointestinal Surgery, 16, 1618-1628. http://dx.doi.org/10.1007/s11605-012-1906-6

[10] Garg, P., Thakur, J.D., Singh, I., Nain, N., Mittal, G. and Gupta, V. (2012) A Prospective Controlled Trial Comparing Single-Incision and Conventional Laparoscopic Cholecystectomy: Caution before Damage Control. Surgical Laparoscopy, Endoscopy \& Percutaneous Techniques, 22, 220-225. http://dx.doi.org/10.1097/SLE.0b013e31824e53db

[11] Cao, Z.G., Cai, W., Qin, M.F., Zhao, H.Z., Yue, P. and Li, Y. (2011) Randomized Clinical Trial of Single-Incision versus Conventional Laparoscopic Cholecystectomy: Short-Term Operative Outcomes. Surgical Laparoscopy Endoscopy \& Percutaneous Techniques, 21, 311-313. http://dx.doi.org/10.1097/SLE.0b013e31822cfacd

[12] Tsimoyiannis, E.C., Tsimogiannis, K.E., Pappas-Gogos, G., Farantos, C., Benetatos, N., Mavridou, P. and Manataki, A. (2010) Different Pain Scores in Single Transumbilical Incision Laparoscopic Cholecystectomy versus Classic Laparoscopic Cholecystectomy: A Randomized Controlled Trial. Surgical Endoscopy, 24, 1842-1848. http://dx.doi.org/10.1007/s00464-010-0887-3

[13] Lirici, M.M., Califano, A.D., Angelini, P. and Corcione, F. (2011) Laparoendoscopic Single Site Cholecystectomy versus Standard Laparoscopic Cholecystectomy: Results of a Pilot Randomized Trial. The American Journal of Surgery, 202, 45-52. http://dx.doi.org/10.1016/j.amjsurg.2010.06.019

[14] Lai, E.C., Yang, G.P., Tang, C.N., Yih, P.C., Chan, O.C. and Li, M.K. (2011) Prospective Randomized Comparative Study of Single Incision Laparoscopic Cholecystectomy versus Conventional Four-Port Laparoscopic Cholecystectomy. The American Journal of Surgery, 202, 254-258. http://dx.doi.org/10.1016/j.amjsurg.2010.12.009 
[15] Phillips, M.S., Marks, J.M., Roberts, K., Tacchino, R., Onders, R., De Noto, G., Rivas, H., Islam, A., Soper, N., Gecelter, G., Rubach, E., Paraskeva, P. and Shah, S. (2012) Intermediate Results of a Prospective Randomized Controlled Trial of Traditional Four-Port Laparoscopic Cholecystectomy versus Single-Incision Laparoscopic Cholecystectomy. Surgical Endoscopy, 26, 1296-1303. http://dx.doi.org/10.1007/s00464-011-2028-Z

[16] Lee, P.C., Lo, C., Lai, P.S., Chang, J.J., Huang, S.J., Lin, M.T. and Lee, P.H. (2010) Randomized Clinical Trial of Single-Incision Laparoscopic Cholecystectomy versus Minilaparoscopic Cholecystectomy. British Journal of Surgery, 97, 1007-1012. http://dx.doi.org/10.1002/bjs.7087

[17] Aprea, G., Coppola Bottazzi, E., Guida, F., Masone, S. and Persico, G. (2011) Laparoendoscopic Single Site (LESS) versus Classic Video Laparoscopic Cholecystectomy: A Randomized Prospective Study. Journal of Surgical Research, 166, 109-112. http://dx.doi.org/10.1016/j.jss.2010.11.885

[18] Blinman, T. (2010) Incisions Do Not Simply Sum. Surgical Endoscopy, 7, 1746-1751. http://dx.doi.org/10.1007/s00464-009-0854-Z

[19] Leung, D., Yetasook, A.K., Carbray, J., Butt, Z., Hoeger, Y., Denham, W., Barrera, E. and Ujiki, M.B. (2012) SingleIncision Surgery Has Higher Cost with Equivalent Pain and Quality-of-Life Scores Compared with Multiple-Incision Laparoscopic Cholecystectomy: A Prospective Randomized Blinded Comparison. Journal of the American College of Surgeons, 215, 702-708. http://dx.doi.org/10.1016/j.jamcollsurg.2012.05.038

[20] Ma, J., Cassera, M.A., Spaun, G.O., Hammill, C.W., Hansen, P.D. and Aliabadi-Wahle, S. (2011) Randomized Controlled Trial Comparing Single-Port Laparoscopic Cholecystectomy and 4-Port Laparoscopic Cholecystectomy. Annals of Surgery, 254, 22-27. http://dx.doi.org/10.1097/SLA.0b013e3182192f89

[21] Saad, S., Strassel, V. and Sauerland, S. (2013) Randomized Clinical Trial of Single-Port, Minilaparoscopic and Conventional Laparoscopic Cholecystectomy. British Journal of Surgery, 100, 339-349. http://dx.doi.org/10.1002/bjs.9003

[22] Trastulli, S., Cirocchi, R., Desiderio, J., Guarino, S., Santoro, A., Parisi, A., Noya, G. and Boselli, C. (2013) Systematic Review and Meta-Analysis of Randomized Clinical Trials Comparing Single-Incision versus Conventional Laparoscopic Cholecystectomy. British Journal of Surgery, 100, 191-208. http://dx.doi.org/10.1002/bjs.8937 